\title{
Single Storage Semi-Global Matching for Real Time Depth Processing
}

\author{
Prathmesh Sawant ${ }^{1}$, Yashwant Temburu ${ }^{1}$, Mandar Datar ${ }^{1}$, Imran Ahmed ${ }^{2}$, Vinayak Shriniwas ${ }^{2}$ and Sachin Patkar $^{1}$ \\ ${ }^{1}$ Department of Electrical Engineering, Indian Institute of Technology Bombay, India \\ ${ }^{2}$ Defence Research and Development Organisation, India
}

\begin{abstract}
Depth-map is the key computation in computer vision and robotics. One of the most popular approach is via computation of disparity-map of images obtained from Stereo Camera. Semi Global Matching (SGM) method is a popular choice for good accuracy with reasonable computation time. To use such compute-intensive algorithms for real-time applications such as for autonomous aerial vehicles, blind Aid, etc. acceleration using GPU, FPGA is necessary. In this paper, we show the design and implementation of a stereo-vision system, which is based on FPGA-implementation of More Global Matching(MGM) [7]. MGM is a variant of SGM. We use 4 paths but store a single cumulative cost value for a corresponding pixel. Our stereo-vision prototype uses Zedboard containing an ARMbased Zynq-SoC [10], ZED-stereo-camera / ELP stereo-camera / Intel RealSense D435i, and VGA for visualization. The power consumption attributed to the custom FPGA-based acceleration of disparity map computation required for depth-map is just $\mathbf{0 . 7 2}$ watt. The update rate of the disparity map is realistic 10.5 fps.

Index Terms-Semi Global Matching(SGM), More Global Matching(MGM), Field Programmable Gate Array(FPGA), System on Chip(SoC), Zedboard, Census Transform, High Level Synthesis(HLS)
\end{abstract}

\section{INTRODUCTION}

Although 2D and 3D LIDARs (Light Detection and Ranging Sensors) provided accuracy, they did not succeed with the economics of power and bill of materials for portable goods. Stereo cameras cost less, but need a lot of computational processing, and this aspect is getting good attention of research community, spurring the development of FPGA and GPU based acceleration of stereo-vision related computation. The low power consumption of fpga-based solutions are attractive and crucial for high performance embedded computing too.

This paper describes our design and implementation of a real-time stereo depth estimation system with Zedboard [10] (housing ARM-SoC based FPGA) at its center. This system uses Zed stereo camera [16], Intel RealSense D435i [15] or ELP stereo-camera for capturing images. Real-time Raster-Respecting Semi-Global Matching [6] (R3SGM) along with Census Transform are used for disparity estimation. The system takes in real-time data from the cameras and generates a depth image from it. Rectification of the images, as well as stereo matching, is implemented in the FPGA whereas capturing data from USB cameras and controlling the FPGA peripherals is done via application programs which run on the hard ARM processor on Zedboard. Development of the FPGA IP's is done using High-Level Synthesis (HLS) tools. A VGA monitor is interfaced to Zedboard to display the computed depth image in real-time.

Our approach is inspired by R3SGM [6] a hardware implementation of SGM. Table III ( at the later portion of the paper ) shows the comparison of hardware utilization between our approach and [6] which shows ours uses much lesser Hardware Resources and thus having less power consumption. It may be emphasized that we have focused on very low power consumption as well as small form factor that is necessary for drones vision, blind aid etc.

\section{LiterATURE REVIEW}

There has been a lot of research on the topic of disparity map generation dating back to 1980s. [8] reviews most of the works including both software and hardware implementations.

A binocular Stereo Camera estimates disparity or the difference in the position of the pixel of a corresponding location in the camera view by finding similarities in the left and right image. There have been various costs governing the extent of the similarity. Some of them are Sum of Absolute Differences(SAD), Sum of Squared Differences(SSD), Normalized Cross-Correlation and the recent Rank Transform and Census Transforms. They are window-based local approaches where the cost value of a particular window in the left image is compared to the right image window by spanning it along a horizontal axis for multiple disparity ranges. The window coordinate for which the metric cost is the least is selected which gives us the disparity for that corresponding center pixel. From the disparity, the depth value is computed by equation 1 where the baseline is the distance between the optical centers of two cameras.

$$
\text { Depth }=\text { Baseline } *(\text { FocalLength }) / \text { disparity }
$$

Local window-based approaches suffer when the matching is not reliable which mostly happens when there are very few features in the surrounding. This results in the rapid variations of the disparities. This problem is solved by global approaches which use a smoothing cost to penalize wide variations in the disparity and trying to propagate the cost across various pixels. The following are some of the global approaches.

\section{A. Semi Global Matching (SGM)}

SGM is a stereo disparity estimation method based on global cost function minimization. Various versions of this 
method (SGM, SGBM, SGBM forest) are still among the top-performing stereo algorithm on Middlebury datasets. This method minimizes the global cost function between the base image and match image and a smoothness constraint that penalizes sudden changes in neighboring disparities. Mutual information between images, which is defined as the negative of joint entropy of the two images, is used in the paper [3] as a distance metric. Other distance metrics can also be used with a similar effect as has been demonstrated with census distance metric in our implementation. Since we already had a Census Implementation, we used it for our SGM implementation. The Hamming Distance returned by Census stereo matching is used as the matching cost function for SGM. The parameters for Census are window size $7 \times 7$, disparity search range 92 . The image resolution is $640 \times 480$. Sum of Absolute Differences (SAD) was also considered as a matching cost function. But it was observed that SAD implementation consumes more FPGA resources than the Census implementation with same parameters. This may be due to the fact that SAD computation is an arithmetic operation whereas Census computation is a logical operation.

Simple census stereo matching has a cost computation step in which for a particular pixel we generate an array of costs (Hamming distances). The length of this array is equal to the disparity search range. The next step is cost minimization in which the minimum of this array (minimum cost) is computed and the index of the minimum cost is assigned as disparity. In SGM, an additional step of cost aggregation is performed between cost computation and cost minimization. The aggregated cost for a particular pixel $\mathrm{p}$ for a disparity index $\mathrm{d}$ is given by equation 2 .

$$
\begin{array}{r}
L_{r}(p, d)=C(p . d)+\min \left(L_{r}(p-r, d),\right. \\
L_{r}(p-r, d-1)+P_{1}, \\
L_{r}(p-r, d+1)+P_{1}, \\
\left.\min _{i}\left(L_{r}(p-r, i)+P_{2}\right)\right) \\
-\min _{k}\left(L_{r}(p-r, k)\right)
\end{array}
$$

For each pixel at direction $r$, the aggregated cost is computed by adding the current cost and minimum of the previous pixel cost by taking care of penalties as shown in Equation 2 Firstterm $C(p, d)$ is the pixel matching cost for disparity $d$. In our case, it is the Hamming distance returned by Census window matching. It is apparent that the algorithm is recursive in the sense that to find the aggregated cost of a pixel $L_{r}^{\prime}(p$,$) ,$ one requires the aggregated cost of its neighbors $L_{r}^{\prime}(p-r$,$) .$ $P_{1}$ and $P_{2}$ are empirically determined constants. For detailed discussion refer to [3].

\section{B. More Global Matching (MGM)}

As SGM tries to minimize the cost along a line it suffers from streaking effect. When there is texture less surface or plane surface the matching function of census vector may return different values in two adjacent rows but due to SGM, the wrong disparity may get propagated along one of the paths and can result in streaking lines.
MGM [7] solves this problem by taking the average of the path cost along 2 or more paths incorporating information from multiple paths into a single cost. It uses this result for the next pixel in the recursion of Equation 2. The resultant aggregated cost at a pixel is then given by the Equation 3

$$
\begin{array}{r}
L_{r}(p, d)=C(p . d)+1 / n \sum_{x \varepsilon\left\{r_{n}\right\}}\left(\operatorname { m i n } \left(L_{r}(p-x, d),\right.\right. \\
L_{r}(p-x, d-1)+P_{1}, \\
L_{r}(p-x, d+1)+P_{1}, \\
\left.\min _{i}\left(L_{r}(p-x, i)+P_{2}\right)\right) \\
\left.-\min _{k}\left(L_{r}(p-x, k)\right)\right)
\end{array}
$$

where $\mathrm{n}$ has the value depending on the number of paths that we want to integrate into the information of single cost. For example, in Figure 1 a two paths are grouped into 1 so $n$ has value 2 and there are a total of 4 groups. Thus we need to store 4 cost vectors in this case and while updating 1 cost value in the center pixel have to read cost vector of the same group from 2 pixels. Lets say $r=1$ for blue boxes group in Figure 1 $1 a$, while updating the $L_{r}$ for this group of the centre pixel in Equation 3 we have $x$ as left and top pixels. From here on SGM refers to MGM variant of it.

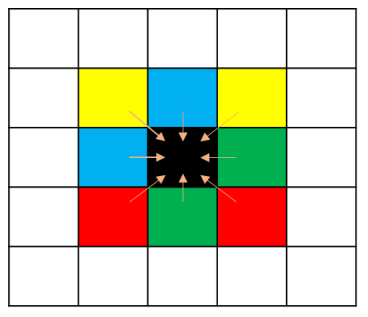

(a) MGM in General

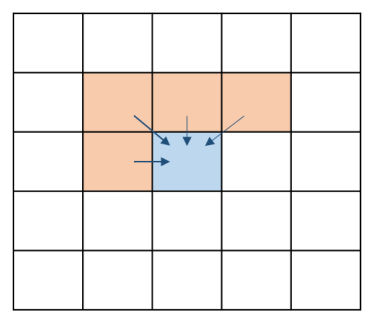

(b) Our Implementation
Fig. 1: Grouping of Paths in MGM

\section{HARDWARE ARCHITECTURE AND IMPLEMENTATION}

\section{A. System Design}

Figure 2 shows an overview of the implemented system. Left and right images captured from the Zed camera [16] are stored into DDR RAM (off-chip RAM). Maps required for the stereo rectification of the images are statically generated offline using OpenCV [17]. These maps are also stored into DDR RAM. We need two Remap peripherals which perform stereo rectification for the left and right images respectively. The Remap peripheral reads the raw image frame and the corresponding map and generates a rectified image frame. The rectified images are again stored into DDR. The Intel RealSense camera requires USB3.0 or higher to stream left and right images. However, Zedboard does not have USB3.0. Hence the camera cannot be directly interfaced to the board. So images were continuously captured and streamed from a computer using ethernet. The left and right image streams were received by a socket client running on the ARM processor on Zedboard. The camera outputs rectified images, hence remap 
peripheral is not required in this case. The images received from the socket client are stored into DDR RAM. We have also implemented it for Zed Camera [16]. For both camera modules in Binocular cameras, the stereo matching peripheral (SGM block in the figure 2) then reads the left and right rectified frame and generates disparity image which is again stored into DDR. The VGA peripheral is configured to read and display the disparity image onto a VGA monitor. FPGA peripherals perform memory access using the AXI4 protocol.

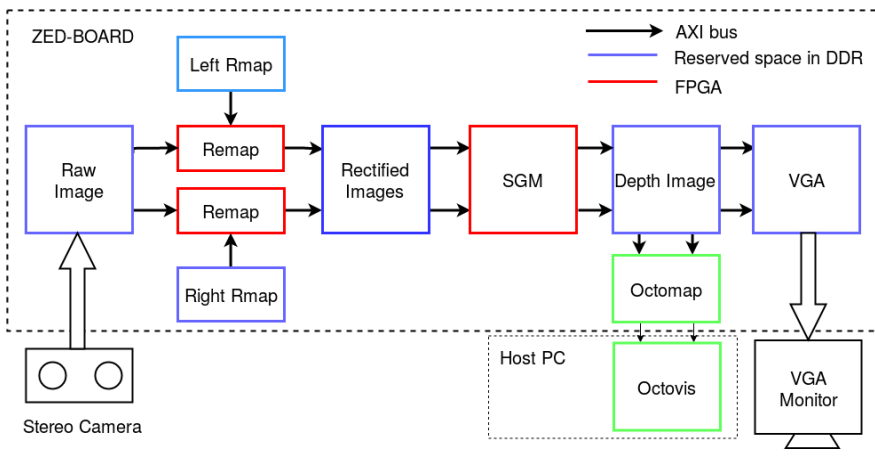

Fig. 2: Block diagram

The resolution of images is fixed to $640 \times 480$ and cameras are configured accordingly. Each pixel is stored as an eight-bit number. The metric used to profile the computation times of different peripherals and also the cameras is fps (frames per second). From here on a frame means 640x480 pixels.

We could have skipped storing the rectified images and passed the output of the Remap peripheral directly to the stereo matching peripheral. We chose not to do this because our performance is not limited by memory read-write but by the FPGA peripherals themselves. We use the AXI4 protocol to perform memory read-write. The read-write rates are 3 orders of magnitude greater than the compute times of FPGA peripherals.

The images are captured using application programs running on the ARM processor on Zedboard. The programs make use of v412 library for image capture. The ARM processor is also used to control the FPGA peripherals.

\section{B. Undistortion and Rectification}

Stereo camera calibration and rectification (one time step) is done using the OpenCV library. Calibration and rectification process produces distortion coefficients and camera matrix. From these parameters, using the OpenCV library, two maps are generated, one for each camera. Size of a map is the same as image size. Rectified images are built by picking up pixel values from raw images as dictated by the maps. The map entry $(i, j)$ contains a coordinate pair $(x, y)$; and the $(i, j)$ pixel in the rectified image gets the value of the pixel at $(\mathrm{x}, \mathrm{y})$ from the raw image. $x$ and $y$ values need not be integers. In such a case, linear interpolation is used to produce final pixel value. Figure 3 shows the remap operation with 4 neighbour bilinear interpolation.

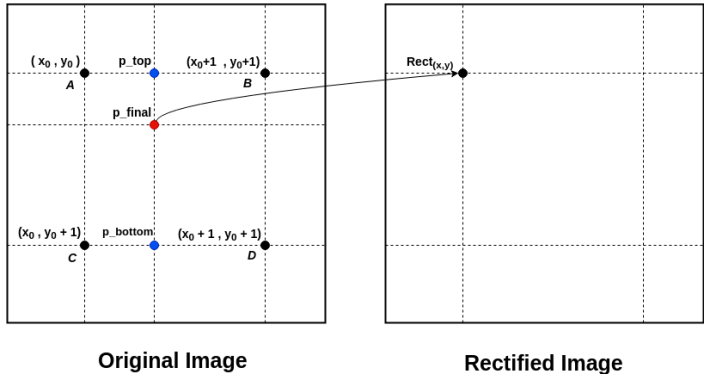

Fig. 3: Remap operation

On-chip memory is limited in size, and it is required by the stereo-depth hardware module. So, we store the maps generated during calibration and rectification in system DDR. The map entries are in fixed-point format with five fractional bits. Captured images are stored in DDR too. The hardware module iterates over the maps, and builds up the result (left and right) images by picking pixels from raw images. Note that, while the maps can be read in a streaming manner, the random-access is required for reading the raw images. For fractional map values, bilinear interpolation (fixed point) is performed. Resulting images are stored back in DDR. As this hardware module has to only - "read maps and raw images pixels from DDR, perform bilinear interpolation, and store the pixels back", it needs less than 5\% resources of the Zynq chip.

\section{SGM Block Architecture}

In Census implementation we scan using row-major order through every pixel in the image and perform stereo matching. Thus for the SGM implementation built upon this, we consider only four neighbors for a pixel under processing as shown in red in Figure 4. This is done because we have the required data from neighbors along these paths. The quality degradation by using 4 paths instead of 8 paths is $2-4 \%$ [4]. Figure 5 shows

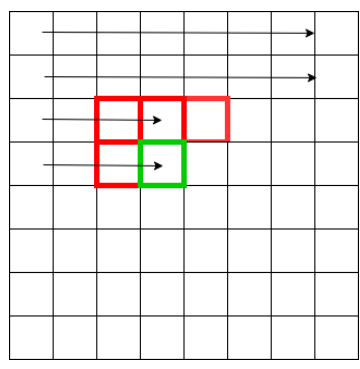

Fig. 4: Four neighbour paths considered for SGM

the implemented SGM architecture. The aggregated cost for all paths and disparity indices of one row above the pixel (full row not shown in figure) and the left adjacent pixel of the current pixel are depicted as columns of colour yellow, red, blue and green for paths top left, top, top right and left respectively.We store the resultant accumulated cost which is computed using Equation 3. 4 Paths have been used by grouping them into single information as shown in Figure 1p. Thus in Equation 3 our $n$ value in 4 and $r$ has a single 
value for a pixel. The Census metric cost is stored in an 8bit unsigned char so the total size of memory occupied by the cost is given as Sizeof RowCostArray $=($ ImageWidth $) *$ $($ DisparityRange $) *($ Noof PathGroups $)=640 * 92 * 1=$ $57.5 \mathrm{~KB}$.

Minimum cost across disparity search range is computed once and stored for the above row and left adjacent pixel. These scalar quantities are shown as small boxes of the same color. Since the minimum cost values are accessed multiple times, storing the minimum values instead of recomputing them every time they are required saves a lot of computations. The pixels in the row above the current pixel can be either topleft, top or top-right neighbors of the current pixels. Hence costs along the left path (green columns) are not stored for the row above the pixel. Figure 5 also shows the data required

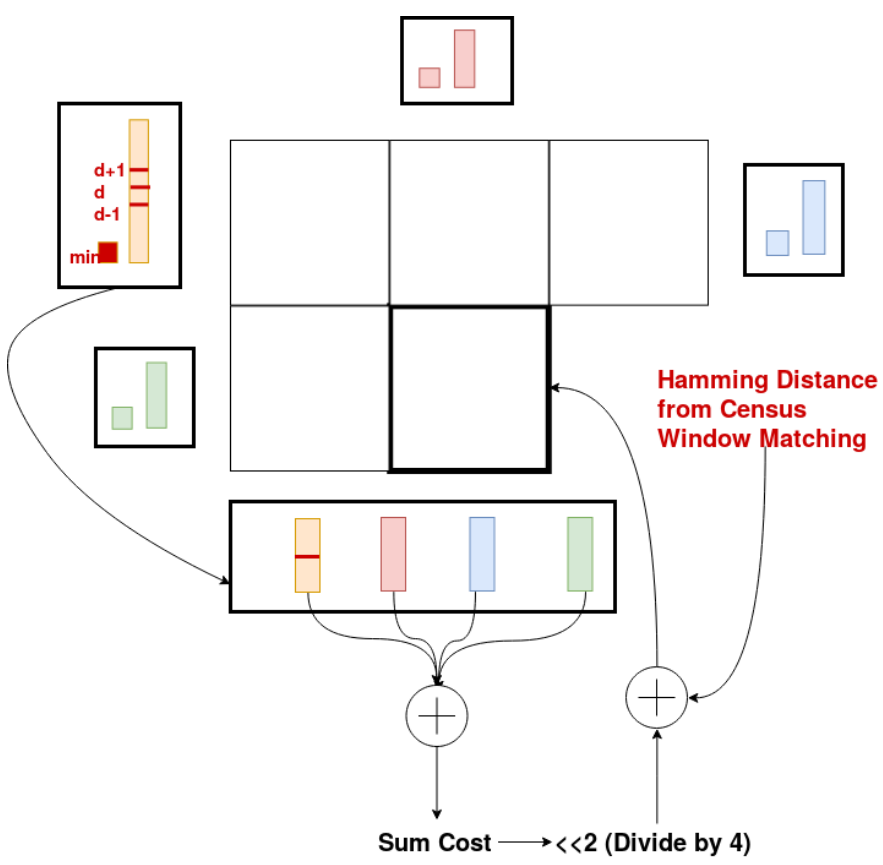

Fig. 5: SGM Cost Computation. Steps involved in calculating the disparity for the current pixel.

and the steps for computing the aggregated cost for a certain pixel considering all the 4 paths. Smoothing term(2nd part in the RHS of Equation 3) along all paths are summed up to obtain a sum cost which has to be divided by $\mathrm{n}(4)$. Since division is resource-intensive hardware we use left a shift by 2 to divide by 4 . Then the resulting value is added with the current hamming distance (1st part in the RHS of Equation 3,. An upper bound is applied to the sum cost. The index of the minimum of this modified sum cost is the disparity for this pixel. The costs for all disparities are stored as they will be required for future pixels of the next row. The minimum cost across the disparity search range is also computed and stored for all paths.

Figure 6 shows the data structures used for storing the costs and the algorithm for updating them as we iterate over pixels. The cost_row structure has dimensions- image columns, path

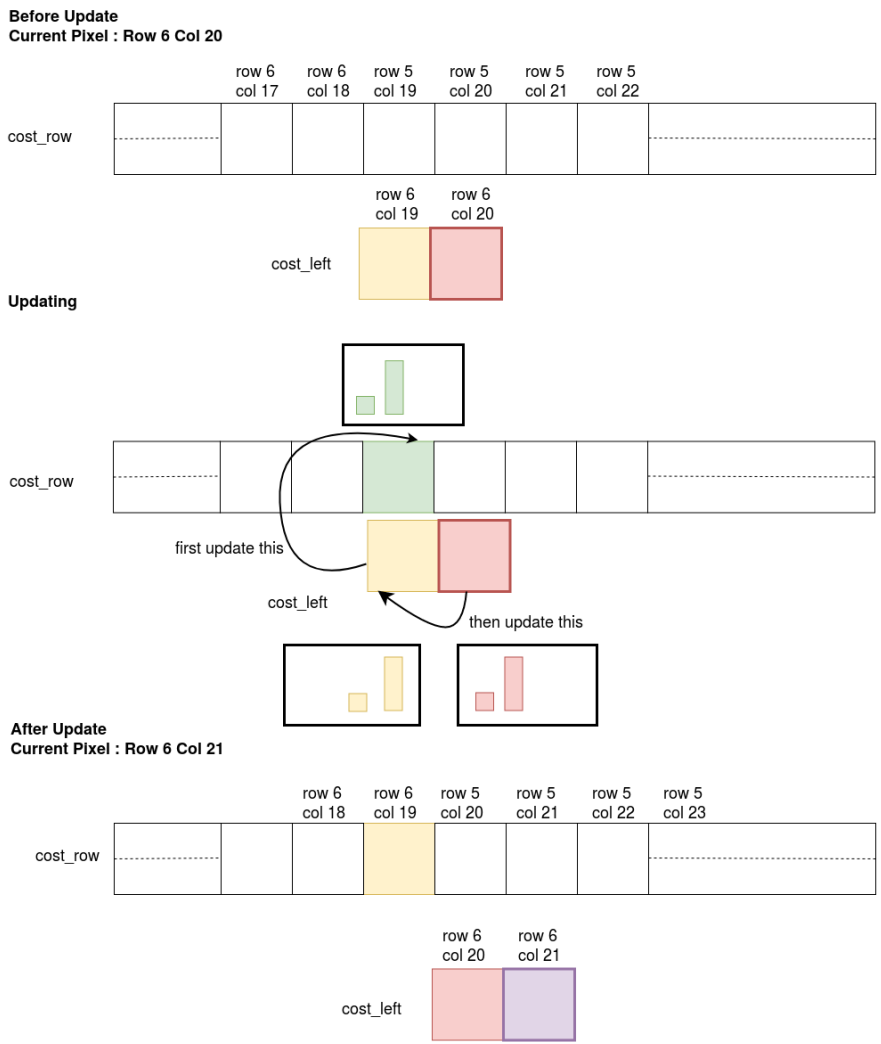

Fig. 6: SGM Array Updation.

groups and disparity search range. It stores the costs for one row above the current pixel for all paths and disparity indices. The cost_left structure has dimensions- path groups and disparity search range. It stores the cost for the left adjacent pixel of the current pixel for all paths and disparity indices. As shown in Figure 6 the current pixel under processing is at row 6 column 20. It requires data from its 4 neighbors: row 5 column 19, row 5 column 20, row 5 column 21 and row 6 column 19.To generate data for current pixel we use the data of cost_left and 3 pixel vectors of cost_row. As we compute the disparity for this pixel and also performing the housekeeping tasks of generating the required data, we update the structures as shown in Figure 6. The data from cost_left is moved to the top-left neighbour of the current pixel in cost_row. The top left pixel cost data is not required anymore and hence is not stored. After this update is done, the currently generated data is moved into cost_left.

Pixels at the top, left and right edge of the image are considered to have neighbors with a maximum value of aggregated cost. As SGM cost aggregation step is a minimization function, they are effectively ignored. The cost_row and cost_left structures are initialized to a maximum value before the stereo matching process. This initialization has to be done for every frame.

\section{HLS Implementation}

High-level Synthesis(HLS) platform such as Vivado HLS ( from Xilinx ) facilitates a suitably annotated description of 
compute-architecture in high level language like $\mathrm{C}$ or $\mathrm{C}++$, which it converts to a low-level HDL based description of the same computing architecture. The generated VHDL or Verilog code is then synthesized to target fpgas. We have used Vivado HLS tools provided by Xilinx to convert our $\mathrm{C}$ implementation to HDL and package it to an IP for further use. The structure of HLS stereo matching code is as follows.

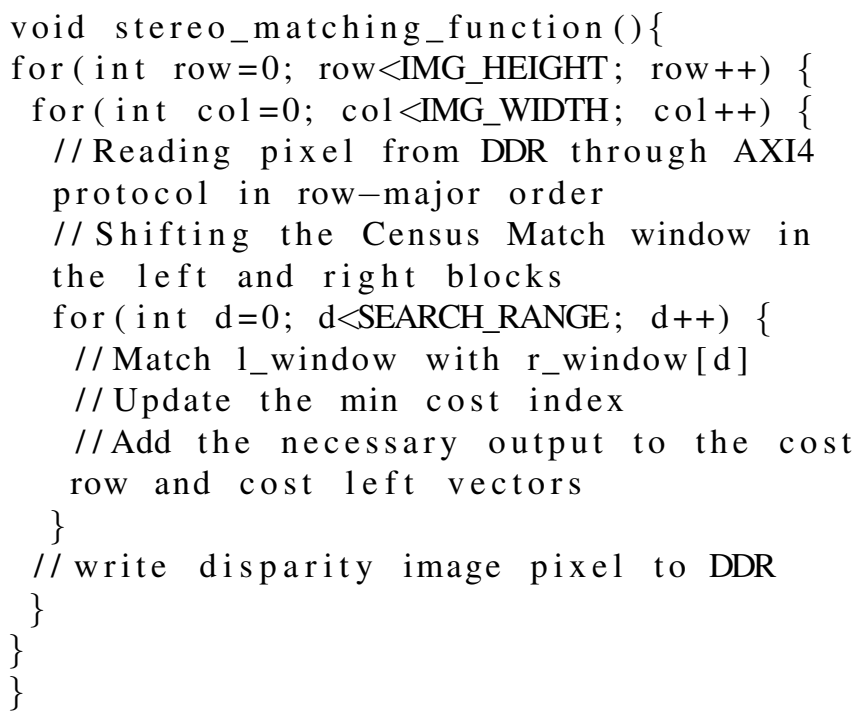

There are no operations between the row and col loop, hence they can be effectively flattened into a single loop. The plan was to pipeline the merged row-column loop. Thus resulting in increase of frame rate by disparity range times if the pipeline throughput had been 1 . However the resources in fpga device on Zedboard are not enough to permit the pipelining the row column loop. Hence, only the search range loop was pipelined. The arrays used in the implementation have been partitioned effectively to reduce the latency. Based on the availability of Hardware resources we have divided the whole image into sections and disparity of each section is computed in parallel. It was observed that a frame rate of $2.1 \mathrm{fps}$ is obtained with the most used resource being Block RAM (BRAM) 17\%. The time required for processing one frame for such an implementation can be given as

$$
\begin{array}{r}
T \propto \text { no. of rows } \times \text { no. of columns } \times \\
(\text { search range }+ \text { pipeline depth })
\end{array}
$$

The characteristic of this implementation is that the logic synthesized roughly corresponds to the matching of two Census windows, the cost aggregation arithmetic and on-chip memory to store data for the next iterations. As we sequentially iterate over rows, columns and disparity search range we reuse the same hardware. Thus, the FPGA resources required are independent of the number of rows, columns and search range but computation time required is proportional to these parameters as shown by equation 4 . This gives us the idea to divide the images into a number of sections along the rows and process the sections independently by multiple such SGM blocks. As the most used resource is BRAM at $17 \%$, we can fit 5 such SGM blocks with each block having to process
5 sections of the image i.e. 128 rows in parallel. Thus we increase resource usage 5 times and reduced the time required for computation by the same resulting in $10.5 \mathrm{fps}$.

One flaw to this approach is that if we divide the input image into exactly 5 parts, there will be a strip of width window size at the center of the disparity image where the pixels will be invalid. The solution to this is that the height of each section is image_height $/ 5+$ window_size/2. This is shown in Figure 7 for an example of 2 sections.
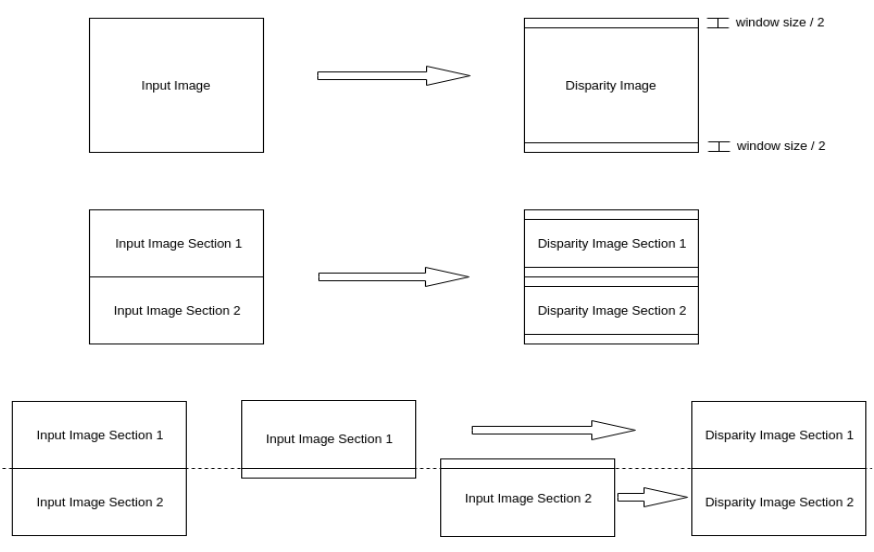

Fig. 7: Dividing the input image into two sections to be processed by two blocks simultaneously

\section{E. Hardware Setup}

Figure 8 shows the hardware setup. The Zed camera is connected to a USB 2.0 port of the Zedboard. The Zedboard is booted with petalinux through SD card. In the case where Intel RealSense camera is used, we require ethernet to receive the images. The only other connections to Zedboard are the connection to VGA display and power.

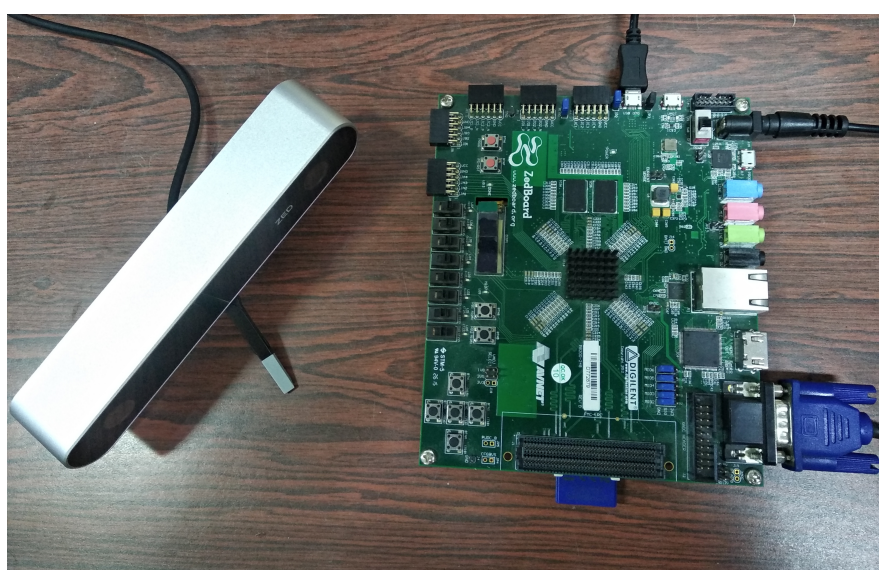

Fig. 8: Hardware setup

\section{EXPERIMENTAL RESULTS AND EVALUATION}

The obtained frame rate for the implemented system is $10.5 \mathrm{fps}$ with Zedboard running at $100 \mathrm{MHz}$. The Power consumption of the computation which is performed in FPGA 
is $0.72 \mathrm{~W}$ whereas the on-chip arm processor which is being used to capture the images and start the FPGA peripherals along with the ELP stereo-camera consumes 1.68 watt , thereby raising consumption to $2.4 \mathrm{~W}$. A $10 \mathrm{~m} \Omega, 1 \mathrm{~W}$ current sense resistor is in series with the $12 \mathrm{~V}$ input power supply on the Zedboard. Header J21 straddles this resistor to measure the voltage across this resistor for calculating Zedboard power [10]. The resource usage is summarized in Table I] It is observed that the BRAM utilization is the most. This is due to storing large cost arrays.

\begin{tabular}{|c|c|c|c|c|c|}
\hline & BRAM & DSP & FF & LUT & LUTRAM \\
\hline Utilization & 132 & 65 & 39159 & 37070 & 981 \\
\hline Available & 140 & 220 & 106400 & 53200 & 17400 \\
\hline \% Utilization & 94.3 & 29.5 & 36.8 & 69.6 & 5.64 \\
\hline
\end{tabular}

TABLE I: Resource utilization for the entire design in Zedboard

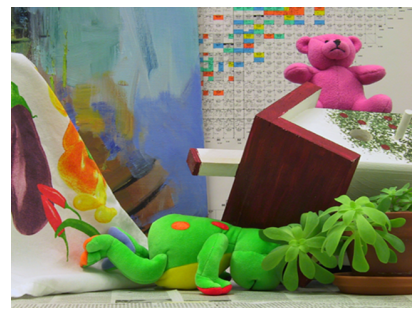

(a) Left image

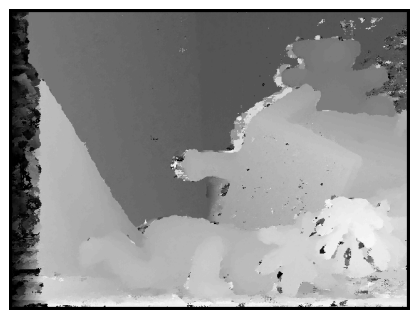

(c) SGM 4 paths software

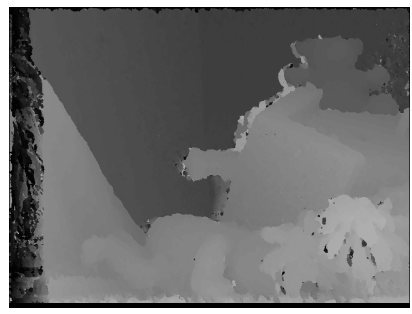

(e) SGM 4 paths hardware

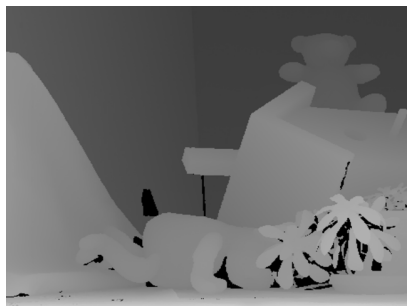

(b) Ground truth

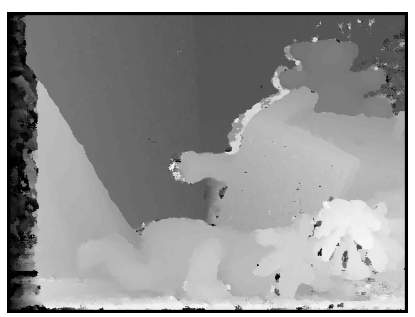

(d) SGM 8 paths software

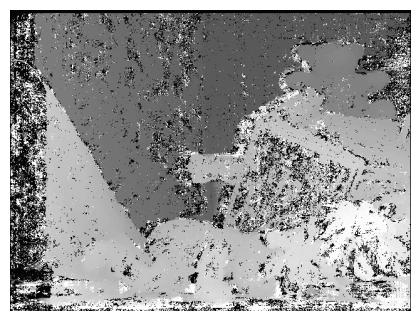

(f) SGM with arrays initialized to zeros
Fig. 9: SGM results on Middlebury images

The algorithmic accuracy is measured using Root mean square of difference in the disparity values obtained by our implementation with the ground truth on Middlebury test images given in Table II column 2. It can also be measured by percentages of erroneous disparities in Table II column 3. A 5 pixel tolerance is considered due to intensity variation caused

\begin{tabular}{|c|c|c|}
\hline Image & RMSE & \% Erroneous disparities \\
\hline Teddy & 5.43 & 11 \\
\hline Dolls & 6.79 & 17 \\
\hline Books & 6.82 & 20 \\
\hline Moebius & 7.54 & 20 \\
\hline Laundry & 9.22 & 27 \\
\hline Reindeer & 9.17 & 27 \\
\hline Art & 9.24 & 30 \\
\hline
\end{tabular}

TABLE II: Accuracy metric of ours disparity image pixels as compared to ground truth for Middlebury images

by changing resolution of raw image. It is notable that no post processing has been done on the SGM output.

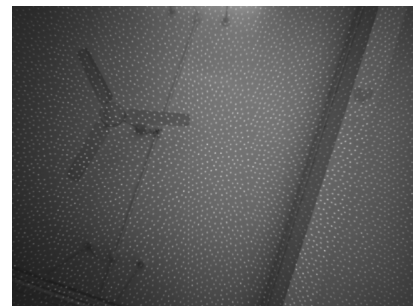

(a) Left image with IR blaster on

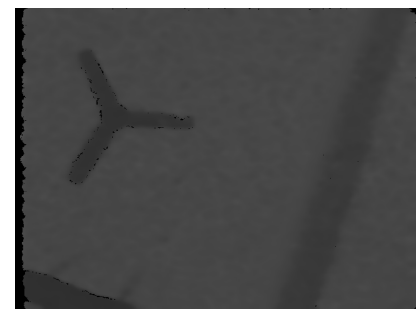

(c) Disparity image from camera with blaster on

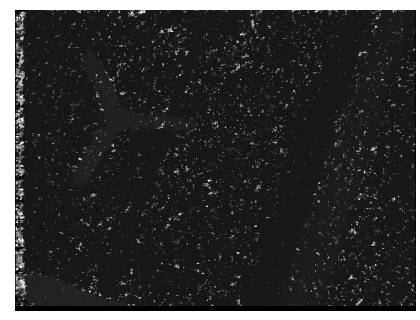

(e) SGM disparity image with blaster on

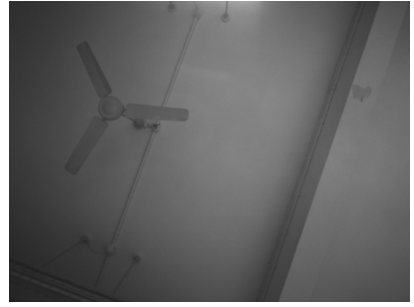

(b) Left image with IR blaster covered

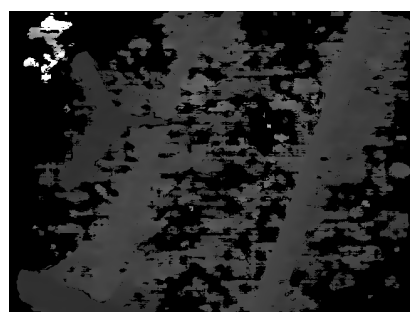

(d) Disparity image from camera with IR blaster covered

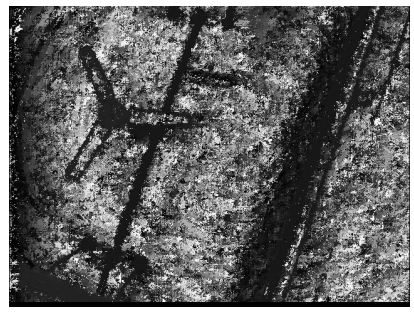

(f) SGM hardware disparity image with IR blaster covered
Fig. 10: SGM results on Realsense image: effect of texture

Figure 9 shows the software and hardware implementation results on Teddy image from Middlebury 2003 dataset [5]. Figure $9 \mathrm{k}$-d show the results of an inhouse software implementation of SGM and Figure 9 9 shows the result of the hardware implementation. It can be observed that SGM with 8 paths gives the best results. SGM with 4 paths in software gives slightly better results than the hardware implementation. The difference in results is due to the fact that the way the algorithm is implemented in software and hardware is different. Figure 9f shows the SGM disparity image with 

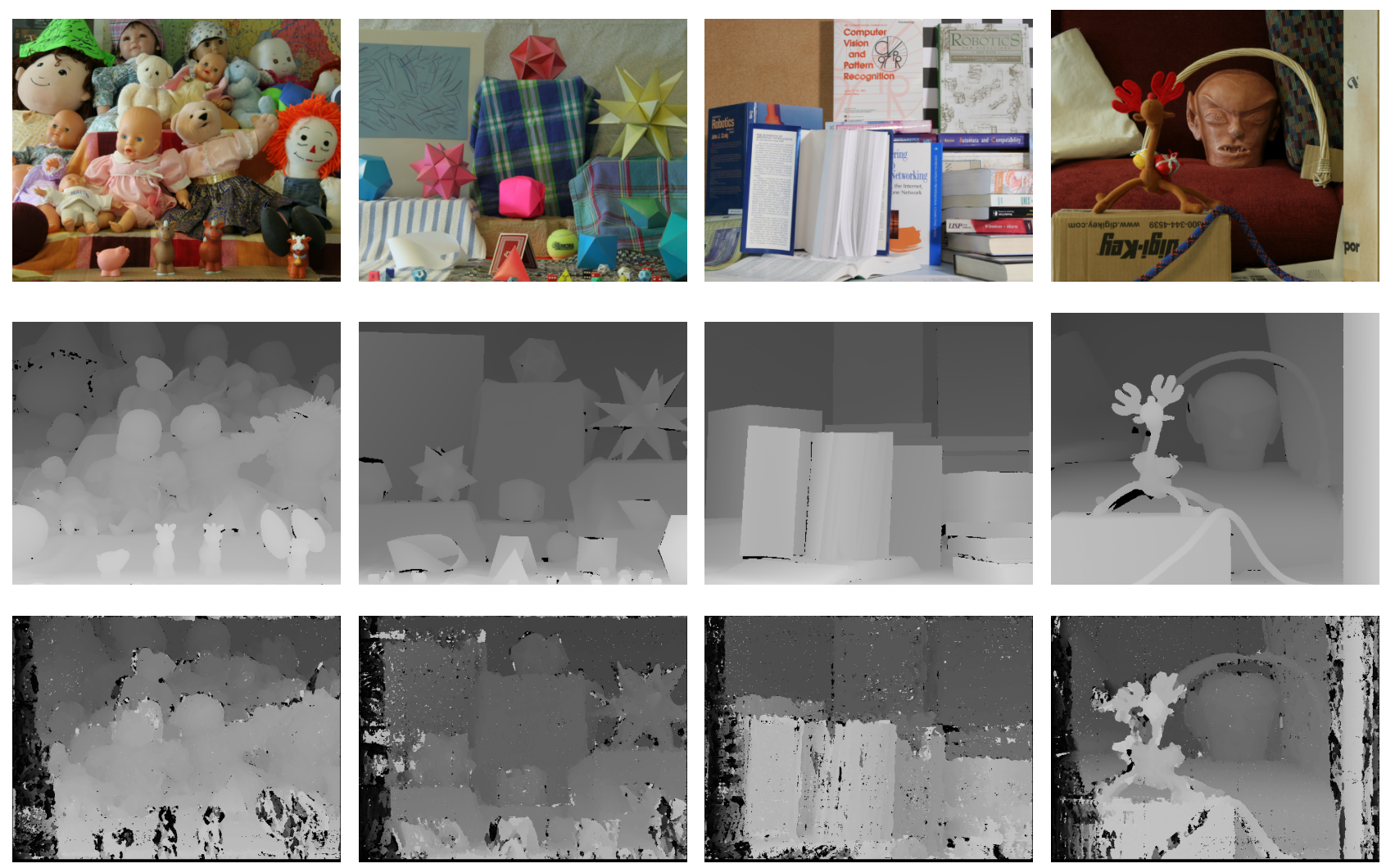

Fig. 11: Qualitative Comparison of our results with some of the Middlebury data set. 1st Row contains the Left Raw Images, 2nd Row contains the ground truth of the corresponding Images and 3rd Row contains the Output of our Implementation.

cost_row and cost_left initialized to zero. Since the cost aggregation function is minimization function, the zeros from the arrays propagate to further pixels. The trickle down effect causes the degradation of the disparity image. Similar results with frame rate around 8.3 fps were also achieved by an inhouse GPU implementation of SGM on Jetson TK1 board which is of MAXWELL architecture with 256 cores and power consumption $<10$ watts. This implementation is analyzed and optimized by using OpenMP for multi-threading and AVX (Advanced Vector Extension) registers for vectorization. GPU shared memory is used to reduce the global memory access. CUDA shuffle instructions are used to speed-up the algorithm and vector processing is also applied.

Fig 12 and 13 shows the captured image and the corresponding disparity image obtained using the SGM implementation. The Intel RealSense camera also provides a disparity image. This is shown in Figure 13b. The convention followed here is opposite i.e closer objects appear darker.

The Intel RealSense camera has an infrared (IR) light projector which projects structured light onto the scene. This pattern can be seen in Figure 13. Figure 10 shows the effect of the infrared projector on disparity estimation. Figure 10 ace show the captured left image from the camera, disparity image obtained from the camera and the computed disparity image when IR blaster was on. Figure 10 pdf show the same images

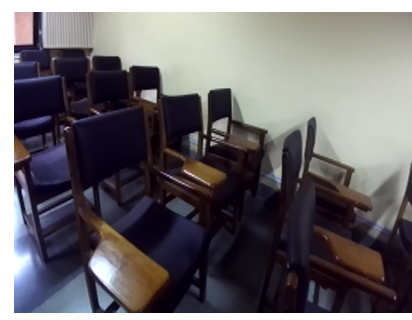

(a) Left image classroom

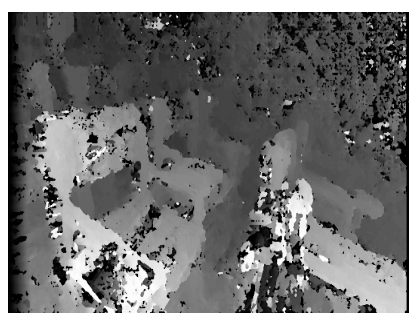

(b) SGM disparity image classroom
Fig. 12: SGM results on ZED camera image

when the IR blaster was covered. Incase of 10 e although the image contains salt noise, it can be easily filtered out. The fan blades can be easily seen in the disparity image. In 10 there are more number of white pixels which imply that the object is very near to the camera which is a false result. As can be seen, the structured light projector helps in stereo matching by adding texture to non-textured surfaces.

Figure 14 shows the scene and the corresponding disparity image obtained on the VGA monitor. The camera can be seen on the left side of the image.

Figure 11 shows the qualitative comparison or our results with Middlebury data set. We can see that the objects placed near are not accurate this is because we have used the disparity 


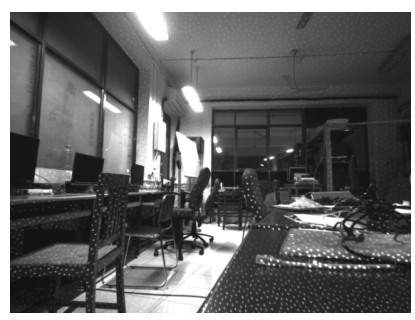

(a) Left image

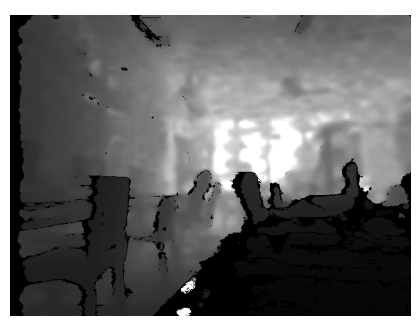

(b) disparity image from the camera

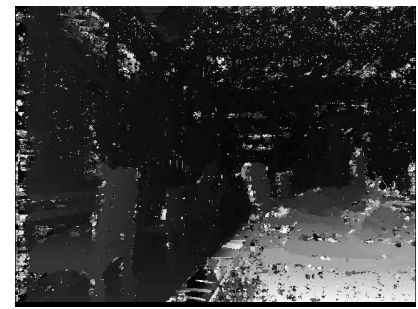

(c) SGM hardware disparity image

Fig. 13: SGM results on Realsense image: lab

range of 92 pixels and so it is not able to find a match in the corresponding left and right images. Thus for a better accuracy, disparity range can be increased with the trade-off being update rate as the pipeline latency will increase.

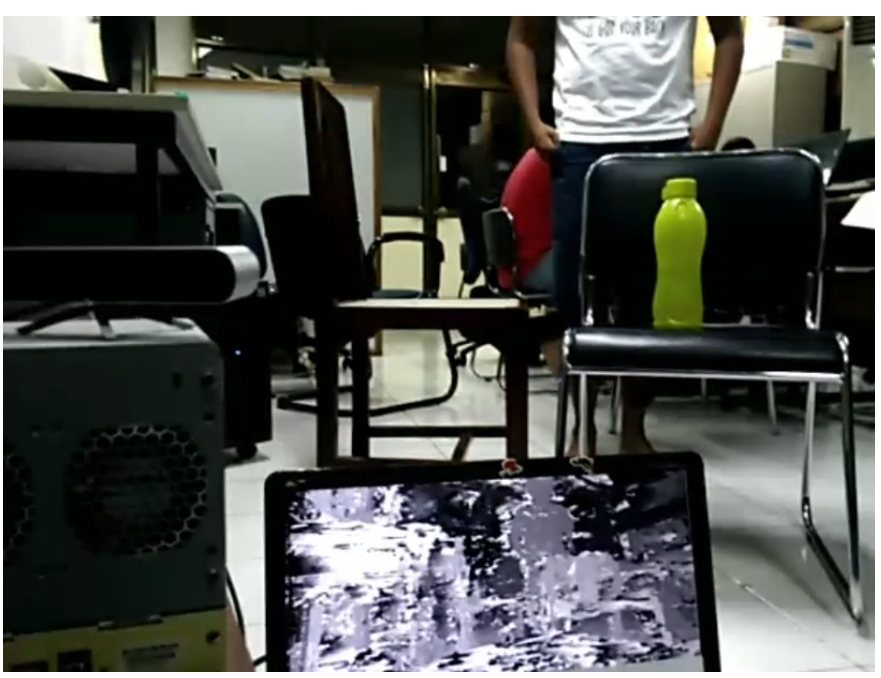

Fig. 14: Scene and disparity image on VGA monitor

Finally we inform the reader about our comparison with R3SGM [6] work. Table III] shows the comparison of hardware utilization between our approach and [6] which shows ours uses much lesser Hardware Resources and thus having less power consumption. Furthermore, if we were to use fpga used in [6], we would have far more liberty with resources that can be leveraged to further pipeline the design and obtain another order of speedup. However we have focused on very low power consumption as well as small form factor that is necessary for drones vision, blind aid etc. We can extrapolate the frame rate likely to be achieved by our design on ZC706 board as below. We can replicate the hardware four times (assuming other resources are under limit) to utilize all of the BRAM, and get 40fps performance. However, it would increase the power consumed by zynq chip, as well as by camera and DDR subsystems for this higher frame capture and processing rate.

\begin{tabular}{|c|c|c|c|c|l|l|}
\hline & BRAM18K & DSP & FF & LUT & $\begin{array}{l}\text { Frame } \\
\text { Rate }\end{array}$ & $\begin{array}{l}\text { Power } \\
\text { (Approx) }\end{array}$ \\
\hline Ours & 132 & 65 & 39159 & 37070 & 10.5 & $0.72 \mathrm{~W}$ \\
\hline$[\overline{6}]$ & 163 & - & 153000 & 109300 & 72 & $3 \mathrm{~W}$ \\
\hline
\end{tabular}

TABLE III: Comparison of FPGA Hardware Resources(Approx) and power consumption between our approach and [6]

\section{CONCLUSION}

In this paper we presented the hardware implementation of the MGM [7] which is a variant of SGM [3] on Zedboard [10] an FPGA-ARM based SOC inspired by R3SGM [6]. In order to reduce the memory consumption, we have grouped 4 pathsleft, top left, top, and top right, whose pixel data are available while processing as a result of row-major order streaming process. The efficient utilization of hardware resources resulted in a low power consumption of $0.72 \mathrm{~W}$ for data processing on FPGA that computes the Rectification and disparity Map generation and with $1.68 \mathrm{~W}$ for data acquisition from Cameras along with starting the peripherals using the on board ARM processor achieving an update rate of $10.5 \mathrm{~Hz}$ with a good accuracy as was shown in TableII and Figure 11 . This system is highly suitable to be used in micro UAVs, blind Aids or any portable types of equipment with a small form factor and high power constraints.

\section{REFERENCES}

[1] R. Zabih and J. Woodfill [Non-parametric local transforms for computing visual correspondence] In Proc. ECCV, pages 151158, 1994

[2] T. Kanade [Development of a video-rate stereo machine] Proceedings of International Robotics and Systems Conference (IROS'95), Pittsburgh, Pennsylvania, Aug. 5-9, 1995, pp. 95-100.

[3] H. Hirschmuller [Stereo Processing by Semiglobal Matching and Mutual Information] IEEE Trans. Pattern Anal. Mach. Intell., 2008,30, (2), pp. 328341

[4] M. Roszkowski and G. Pastuszak [FPGA design of the computation unit for the semi-global stereo matching algorithm] doi: 10.1109/DDECS.2014.6868796

[5] D. Scharstein and R. Szeliski [High-accuracy stereo depth maps using structured light] IEEE Computer Society Conference on Computer Vision and Pattern Recognition (CVPR 2003), volume 1, pages 195202, Madison, WI, June 2003

[6] Oscar Rahnama, Tommaso Cavallari, Stuart Golodetz, Simon Walker and Philip H. S. Torr . [R3SGM: Real-time Raster-Respecting SemiGlobalMatching for Power-Constrained Systems] International Conference on Field-Programmable Technology (FPT), Vietnam, 2018.

[7] G. Facciolo, C. de Franchis, and E. Meinhardt [MGM: A Significantly More Global Matching for Stereovision.] BMVC, 2015.

[8] Rostam Affendi Hamzah and Haidi Ibrahim [Literature Survey on Stereo Vision Disparity Map Algorithms]vol. 2016, Article ID 8742920, 23 pages, 2016.

[9] W.Daolei, K.B.Lim, [Obtaining depth maps from segment-based stereo matching using graph cuts], J.Vis.Commun. Image R.22 (2011)325-331.

[10] Zedboard datasheet:(2019,August 25) Retrieved from http://zedboard.org/sites/default/files/documentations/ZedBoard HW UG v2 2.pdf 
[11] Zynq 7000 datasheet:(2019,August 25) Retrieved from https://www.xilinx.com/support/documentation/data_sheets/ds190-Zynq -7000-Overview.pdf

[12] Vivado HLS user guide:(2019,August 25) Retrieved from https://www.xilinx.com/support/documentation/sw_manuals/xilinx2014 1/ug902-vivado-high-level-synthesis.pdf

[13] Vivado Synthesis user guide:(2019,August 25) Retrieved from https://www.xilinx.com/support/documentation/sw_manuals/xilinx2017 3/ug901-vivado-synthesis.pdf

[14] XSCT reference guide:(2019,August 25) Retrieved from https://www.xilinx.com/support/documentation/sw_manuals/xilinx2016 _2/ug1208-xsct-reference-guide.pdf

[15] Intel Realsense D435i Depth Camera:(2019,August 25) Retrieved from https://www.intelrealsense.com/depth-camera-d435i/

[16] Zed Camera:(2019,August 25) Retrieved from www.stereolabs.com

[17] OpenCV:(2019,August 25) Retrieved from https://opencv.org/ 